\title{
Peripheral viewing during exposure to a 2D/3D video clip: effects on the human body
}

\author{
Masumi Takada $\cdot$ Yuta Fukui $\cdot$ Yasuyuki Matsuura $\cdot$ \\ Motohiko Sato $\cdot$ Hiroki Takada
}

Received: 30 October 2014/Accepted: 10 November 2014/Published online: 24 December 2014

(C) The Japanese Society for Hygiene 2014

\begin{abstract}
Objectives Symptoms of three-dimensional (3D) sickness, such as intoxication and eye fatigue, have been observed in subjects viewing 3D films and vary according to the image quality and visual environment. In addition, the influence of stereoscopic vision on the incidence of 3D sickness has not been explored sufficiently. Therefore, it is important to examine the safety of viewing virtual 3D content.

Methods The present study examines the effects of peripheral vision on reported motion sickness during exposure to 2D/3D video clips for $1 \mathrm{~min}$ and for $1 \mathrm{~min}$ afterwards in human subjects. Stabilograms were recorded during exposure to video clips with or without visual pursuit of a 3D object and compared, and subjects were administered the simulator sickness questionnaire after stabilometry.

Results There were no significant main effects of solidity of the visual stimulous (2D/3D) and the vision method (visual pursuit/peripheral viewing) in accordance with the two-way analysis of variance of the sway values, although the sway values during the $2 \mathrm{D} / 3 \mathrm{D}$ video clips were higher than in control subjects. A consistent trend in the main effect of stability was observed. Further, the sway values
\end{abstract}

M. Takada $(\bowtie) \cdot$ M. Sato $\cdot$ H. Takada

Department of physiology, Aichi Medical University,

Nagakute, Aichi 480-1195, Japan

e-mail: masumasu@aichi-med-u.ac.jp

Y. Fukui · H. Takada

Department of Human and Artificial Intelligent System,

Graduate School of Engineering, University of Fukui,

3-9-1 Bunkyo, Fukui, Fukui 910-8507, Japan

\section{Y. Matsuura}

Graduate School of Natural Sciences, Nagoya City University,

1 Yamanohata, Mizuho-cho, Mizuho-ku, Nagoya,

Aichi 467-8501, Japan changed remarkably after the 3D video clip was viewed peripherally and produced a persistent instability in equilibrium function. The questionnaire findings also significantly changed after the subjects viewed the video clips peripherally.

Conclusions Subjective exacerbation and deterioration of equilibrium function were observed after peripheral viewing of 3D video clips. This persistent influence may result when subjects view a poorly depicted background element peripherally, which generates depth perception that contradicts daily experience.

Keywords Body sway $\cdot$ Stabilogram $\cdot$ Stereoscopic image $\cdot$ Simulator sickness questionnaire $\cdot$ SSQ

\section{Introduction}

In recent times, the familiarity towards three-dimensional (3D) images has increased greatly because 3D movie contents providing binocular parallax are loaded onto different sources of amusement, such as cinema, home television, and game machines. Although the visual presence has been enhanced by the progress in 3D technology, which enables portrayal of movie scenes more realistically, each merit has its demerit. In this case, motion sickness is induced while viewing stereoscopic video clips [1].

The standing posture in humans is maintained by the body's balance function, which is an involuntary physiological adjustment mechanism called the "righting reflex" [2]. This righting reflex, which is centered in the nucleus ruber, is essential to maintain the standing posture when the body is stationary. For this, the body's balance function utilizes sensory signals such as visual, auditory, and vestibular inputs, as well as proprioceptive inputs from the 
skin, muscles, and joints [3]. The evaluation of this function is indispensable for diagnosing equilibrium disturbances like cerebellar degenerations, basal ganglia disorders, or Parkinson's disease [4]. Since long, stabilometry has been employed for a qualitative and quantitative evaluation of this equilibrium function. In this, a projection of a subject's center of gravity onto a detection stand is measured as an average of the center of pressure (COP) of both the feet. The COP is traced for each time step, and the time series of the projections is traced on an $x-y$ plane (this has been explained in detail in the following section). By connecting the temporally vicinal points, a stabilogram is created. Several parameters are widely used in clinical studies to quantify the degree of instability in the standing posture; for instance, the area of sway $(A)$, total locus length $(L)$, and locus length per unit area $(L / A)$. It has been revealed that the last parameter is particularly related to the fine variations involved in posture control [2]. Thus, the $L / A$ index is regarded as a gauge for evaluating the function of the proprioceptive control of standing in human beings. However, it is difficult to clinically diagnose disorders of balance and identify the decline in the equilibrium function by utilizing the abovementioned indices and measuring patterns in a stabilogram alone. In addition, large inter-individual differences might make it difficult to understand the results of such a comparison. Accordingly, we proposed a new index, called sparse density (SPD) [5, 6], which depends on the density of COPs in some local area of the stabilograms. With reference to the mathematical model of systems to control upright postures, the SPD quantifies the stability of the systems, and is deemed significant (see "Appendix").

Watching 3D movies, though, can produce certain adverse effects such as asthenopia and motion sickness [7]. It has been considered that this visually induced motion sickness (VIMS) is caused by the sensory conflict that results from the disagreement between convergence and visual accommodation while viewing 3D images [8]. Thus, stereoscopic images have been devised to reduce this disagreement $[9,10]$. In this paper, we also examine whether the VIMS is caused by this kind of the sensory conflict.

Visually induced motion sickness (VIMS) can be measured by psychological and physiological methods, and the simulator sickness questionnaire (SSQ) is a well-known psychological method for measuring the extent of motion sickness [11]. The SSQ has been used in the present study to verify the occurrence of VIMS. In addition, the following parameters of autonomic nervous activity are considered appropriate for the physiological method: heart rate variability, blood pressure, electrogastrography, and galvanic skin reaction [12-14]. It has been reported that a wide stance (with the midlines of the heels from 17 to $30 \mathrm{~cm}$ apart) significantly increases the total locus length in the stabilograms of individuals with high SSQ scores, while the length for individuals with low scores is less affected by such a stance [15]. We reported that VIMS could be detected by the total locus length and SPD, which were used as the analytical indices of stabilograms [16].

The recent widespread use of stereoscopic vision facilitates provision of virtual reality and sensation; however, as discussed, since long, there has been concern over the symptoms caused by stereoscopic vision. Accordingly, in the present study, we have examined whether our visual and equilibrium systems are affected by an hour-long session of stereoscopic viewing [17, 18]. In an earlier study by Yoshikawa et al. [18], the sway values measured after visual pursuit of the stereoscopic sphere in a video clip tended to become smaller than those after (peripheral) viewing without purposeful pursuit of the object. In addition, when the subjects were not allowed to fix the point of gaze as they wished, they looked at the objects with different virtual depth. Each time subjects change their point of gaze, interaction occurs between systems to control accommodation and convergence. A state of non-equilibrium in the control system for visual information processing imposes additional load on the human body. Further, the intermittent inconsistency between accommodation and convergence is considered to cause imbalance in our autonomic nervous system. To examine this hypothesis, we measured the severity of the influence of peripheral viewing, which is expected to cause uncomfortable symptoms of motion sickness, and compared it with the influence of the visual pursuit of a virtual object on the equilibrium function. In addition, we discussed the relationship among convergence, accommodation of lenses, and depth of the virtual object observed when subjects complain of the discomfort caused by 3D sickness.

\section{Materials and methods}

Eleven healthy men (mean \pm standard deviation, $22.6 \pm 0.7$ years), who may have had any otorhinolaryngologic or neurological diseases in the past, participated in this study. The experiment was sufficiently explained to the subjects, following which written consent was obtained from them. The research was approved by the Ethics Committee, Nagoya City University Graduate School of Natural Sciences: Project ID Number 42.

In this experiment, the body sway was measured while viewing $2 \mathrm{D} / 3 \mathrm{D}$ video clips. The subjects stood on the detection stand of a stabilometer GS3000 (Anima Co. Ltd., Tokyo), without moving, with their feet together in the Romberg posture, for $30 \mathrm{~s}$ before the sway was recorded. Each sway of the COP was then recorded at a sampling frequency of $20 \mathrm{~Hz}$. The subjects were instructed to 


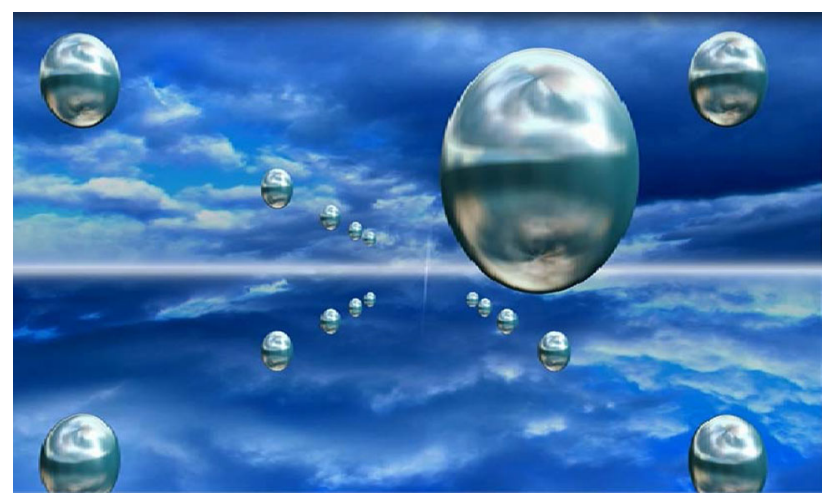

Fig. 1 The Sky Crystal program generates smooth locomotion in the sphere and as subjects view the image, forces lens accommodation to alternate between proximal and distal depths. Performance of the ciliary muscles is enhanced by this accommodation training. Previous studies indicate that low myopia and eye strain can be improved using this strategy $[17,34]$

maintain the Romberg posture during the trials. For the first $60 \mathrm{~s}$, the subjects were asked to do the following:

I. Gaze at a static circle with a diameter of $3 \mathrm{~cm}$ (Control).

II. Follow a sphere which complexly ambulated in a video clip (Fig. 1).

III. Peripherally view the same video clip as shown in (II) without pursuing the sphere on the 40-inch display KDL-40HX80R (Sony, Tokyo).

That is, we categorized the visual sighting method as pursuit (II) and peripheral viewing (III). Subjects gazed at a point of fixation (I) or video clips (II)/(III) with their eyes open for the first $60 \mathrm{~s}$, after which they closed their eyes for $60 \mathrm{~s}$. In addition, subjective evaluation of symptoms caused by the motion sickness was examined using the SSQ and visual analog scale (VAS), which were administered after the stabilometry.

The circle (I) was placed before the subjects, $2 \mathrm{~m}$ away, at their eye level. Stereoscopic video clips (II)/(III) and their monocular (2D) vision were shown to subjects on the binocular parallax 3D display. The content in the "Sky Crystal" (Olympus Memory Works Ltd. Co., Tokyo) was modified, with permission from the company, and used as the visual stimulus in this experiment. The stimulus includes spheres fixed in four corners, which supplies the perspective. We measured the body sway and the subjective evaluation for each vision (I) Control, (II)-2D, (II)3D, (III)-2D, and (III)-3D situation randomly, according to the abovementioned protocol.

Items

We conducted the stabilometry with eyes open/closed. The experimental periods with eyes open and closed were designed in our experimental protocol to evaluate the severity of the VIMS during and after viewing the video clips. In stabilometry, the COP on an $x-y$ plane was recorded at each time step where $x$ and $y$ directions were defined as the right and the anterior planes on their faces, respectively. Stabilograms were obtained each experimental period from the time series of their COP. Finally, we calculated the new index SPD, and the previously stated sway values such as area of sway, total locus length, total locus length per unit area, defined in Suzuki et al. [19].

The abovementioned indices were calculated from each stabilogram recorded with eyes open/closed. In addition, we employed the following statistical analysis for the subjective evaluation of the symptoms of the VIMS. To evaluate simulator sickness, the 16 subjective items of the SSQ were used, which were extracted from 1,119 variable pairs from the Motion Sickness Questionnaire, measured before and after simulator exposure, based on the results of a factor analysis [11]. Nausea (N), Oculomotor Discomfort (OD), Disorientation (D), and Total Score (TS) are known as sub-scores of the SSQ. In the present study, the maximum scores of N, OD, D, and TS were 66.78, 53.06, 97.44, and 78.54 , respectively. In this paper, the significance level was set at $p=0.05$.

The influence of exposure to the video clips on our equilibrium system

The influence of exposure to the video clips on our equilibrium system was investigated in comparison with the control data (I). Friedmann tests were employed for each sway value and index of the stabilogram observed while and after viewing the stimuli in the (I), (II)-2D, (II)-3D, (III)-2D, and (III)-3D conditions. In addition, the Nemenyi method was used for multiple comparisons [20].

Solidity of the subjects' vision and their vision method

Solidity of the subjects' vision (2D/3D) and their vision method (visual pursuit/peripheral viewing) were assumed to be important influencing factors, on which a two-way analysis of variance (ANOVA) was conducted for the number of repetitions 11 . In addition, the sway values while and after viewing the (II)-2D, (II)-3D, (III)-2D, and (III)-3D stimuli were compared using Wilcoxon signedrank tests.

Subjective evaluation

Subjective evaluation was conducted by filling out the questionnaires before and after the stabilometry. In addition to the SSQ, the VAS was utilized to evaluate their eye strain. Finally, a two-way ANOVA on the abovementioned 
factors was conducted for each sub-score, specifically, the $\mathrm{N}, \mathrm{OD}, \mathrm{D}, \mathrm{TS}$, and VAS.

Lens accommodation and convergence distance

The participants in this study were two middle-aged individuals in their thirties to forties and four young subjects (age $23 \pm 1$ years). We obtained informed consent from all participants.

We placed an LCD monitor CINEMA 3D 42LW5700 (LG Electronics, Seoul) facing the participants at a distance of $1.57 \mathrm{~m}(=3 \mathrm{H})$. We presented either a (II)-2D or (II)-3D video clip on the monitor. The abovementioned spherical object appeared as a 3D video clip located at a virtual distance of $1.57 \mathrm{~m}$ and moved toward the participants to a virtual distance of $1.19 \mathrm{~m}$. We asked the participants to gaze at the center of the spherical object for $60 \mathrm{~s}$ and measured their lens accommodation and convergence distance during this experiment. The 3D video clip was presented using a circular polarizing filter system.

We developed an original machine by combining the WAM- $5500^{\circledR}$ and EMR- $9^{\circledR}$ to perform the measurements. The WAM-5500 is an auto refractometer (Grand Seiko Co, Ltd, Fukuyama) that can measure accommodative power under natural conditions when both eyes are open. It can continuously record accommodative focus distance at a rate of $5 \mathrm{~Hz}$. The EMR-9 is an eye mark recorder (NAC Image Tech. Inc., Tokyo) that can measure the convergence distance using the pupillary/corneal reflex method. We used a circular polarizing filter system combined with the respective binocular vision systems to present $2 \mathrm{D}$ and $3 \mathrm{D}$ video clips.

\section{Results}

Typical stabilograms of the subjects following the virtual sphere have been shown in Figs. 2 and 3. With eyes open, most stabilograms observed during peripheral viewing of the $2 \mathrm{D} / 3 \mathrm{D}$ video clips were dispersed as compared to the control stabilograms. In contrast, no consistent tendency was observed in the stabilograms measured when the subjects' eyes were closed. Moreover, most stabilograms observed during and after exposure to the video clips were dispersed in comparison to the control stabilograms.

Influence of the exposure to video clips on our equilibrium system

The sway values, for when the subjects' eyes were open, were calculated from the stabilograms. Except for the total locus length per unit area, the control sway values were smaller than those obtained from stabilograms recorded during exposure to the 2D/3D video clips (Fig. 4). Further, according to the Friedman test $(p<0.05)$, there was a significant difference among the sway value levels of the (I), (II)-2D, (II)-3D, (III)-2D, and (III)-3D conditions. These sway values for the control were significantly smaller than those obtained from stabilograms from conditions involving visual pursuit of the sphere $(p<0.05)$. The sway values for the control also tended to be smaller than those obtained from stabilograms recorded during peripheral viewing of the stereoscopic video clip $(p<0.1)$.

With reference to the results of stabilometry with eyes closed, except for the total locus length, there tended to be a significant difference among sway value levels for the (I), (II)-2D, (II)-3D, (III)-2D, and (III)-3D situations, as verified by the Friedman test $(p<0.1)$. Mainly, the control equilibrium system tended to be more stable than that observed during peripheral viewing of the stereoscopic video clip $(p<0.1)$. This was particularly observed with reference to the area of sway, total locus length per unit area, and SPD $S_{4}$ (Fig. 4d, f). SPD $S_{3}$ had similar results. The sway values for situations involving visual pursuit of the 2D/3D sphere did not differ from the control, whereas the significant influence was seen in the results for when stabilometry was conducted with the subject's eyes open (Fig. 4a, b, c).

\section{Solidity of the subjects' vision and their vision method}

The two-way ANOVA on sway values did not reveal any interaction between the two factors: solidity of the subjects' vision (2D/3D) and their vision method (visual pursuit/peripheral viewing).

With eyes open, the sway values during the exposure to video clips (II)-2D, (II)-3D, (III)-2D, and (III)-3D were compared using the Wilcoxon signed-rank test whereas the main effects were not observed for any sway values. Except for the total locus length, there was no significant difference between any stabilograms observed during the exposure to the abovementioned video clips.

With eyes closed, results of the two-way ANOVA for the area of sway and SPD $\mathrm{S}_{3}, \mathrm{~S}_{4}, \mathrm{~S}_{5}$ showed a consistent main effect of the solidity $(p<0.1)$. According to the Wilcoxon signed-rank test for total locus length per unit area, in addition to these indices, the subjects' equilibrium systems after exposure to the 3D video clip were significantly less stable than that after exposure to the $2 \mathrm{D}$ one, involving peripheral viewing of the video clips. Moreover, the same nonparametric test for the total locus length measured after exposure to the $3 \mathrm{D}$ video clip was slightly smaller than those measured after exposure to the $2 \mathrm{D}$ one (Fig. 4e). 
(a)

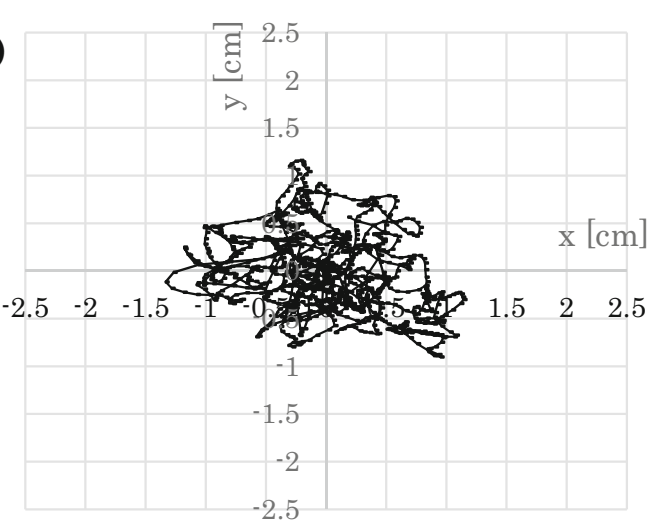

(b)

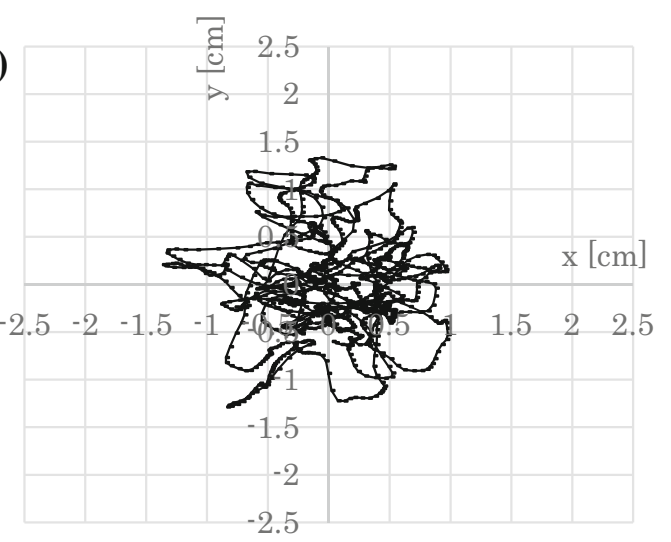

(c)

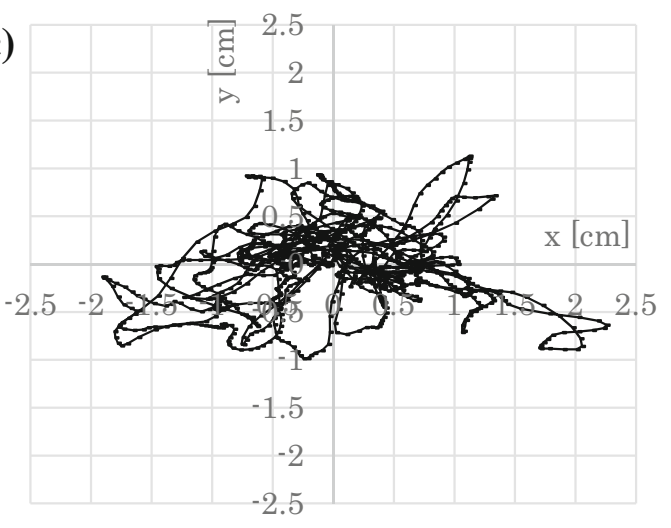

(d)

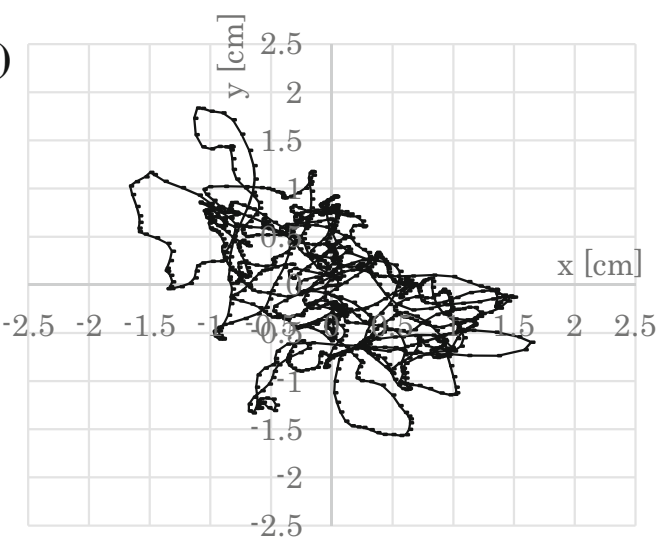

(e)

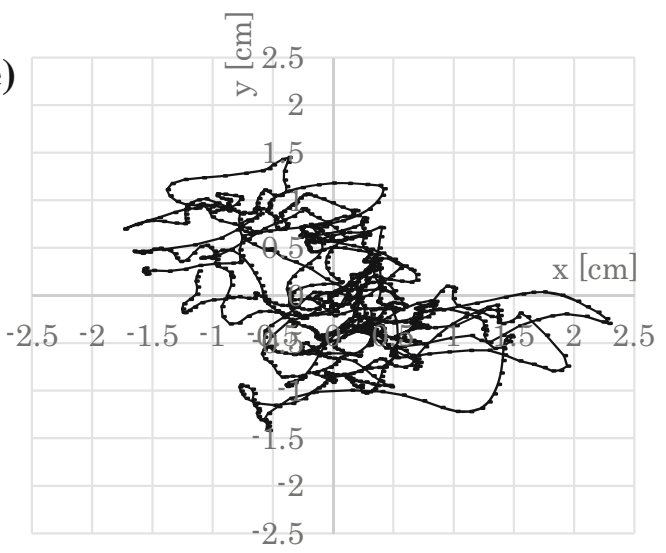

Fig. 2 Representative stabilograms recorded with the eyes open. The control stabilogram (a) was compared to stabilograms recorded while subjects peripherally viewed 2D (b) and 3D (c) video clips, and while visually tracking 2D (d) and 3D (e) video clips

Subjective evaluation

The SSQ confirmed that the subjects did not complain of discomfort before the stabilometry and after the control stabilometry. Any sub-scores of the SSQ filled out after the exposure to the $3 \mathrm{D}$ video clip were greater than those filled after the exposure to the 2D one. Moreover, any sub-scores of the SSQ after peripheral viewing of the video clips were also greater than those measured after exposure to the visual pursuit ones (Fig. 5).

According to the two-way ANOVA, with reference to the subjective indices, interaction was not found between the abovementioned factors. In the sub-scores for N, OD, and TS, the main effect of the vision method was observed 
(a)

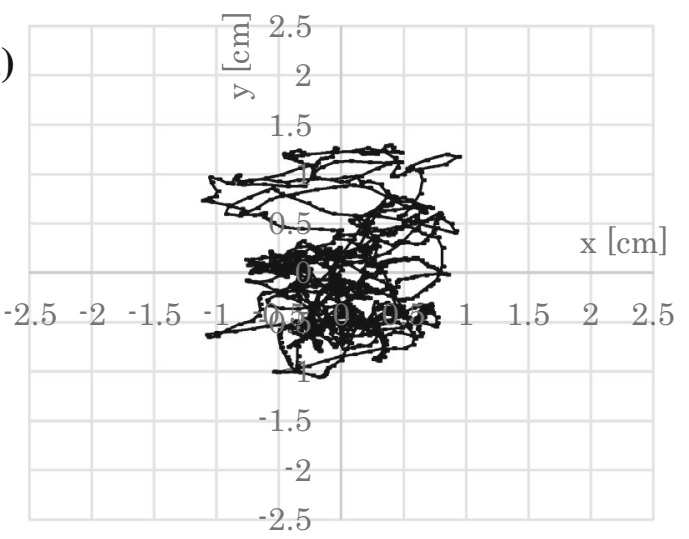

(b)

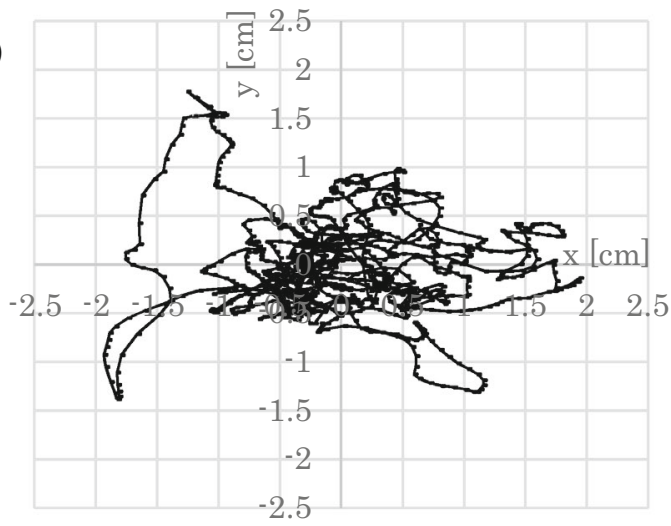

(c)

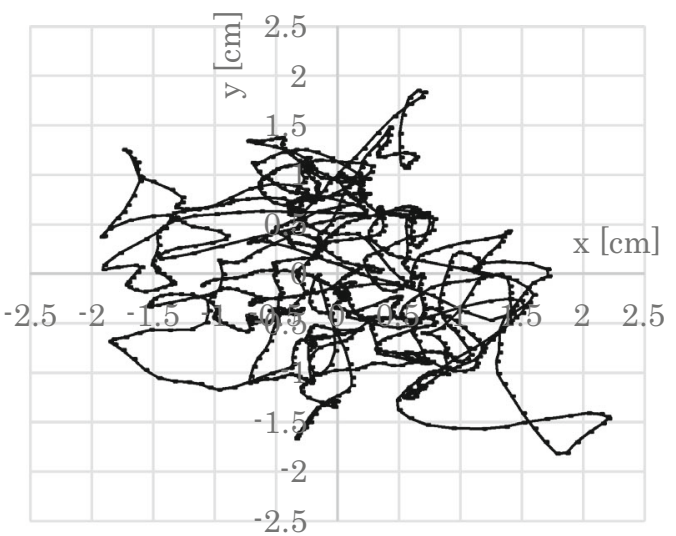

(d)

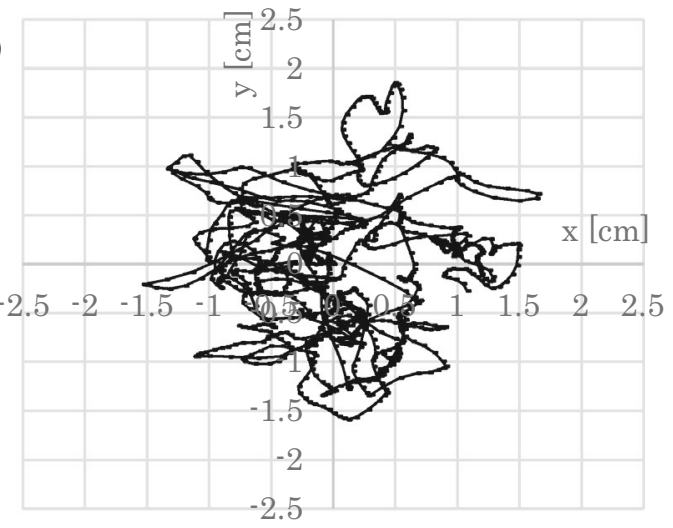

(e)

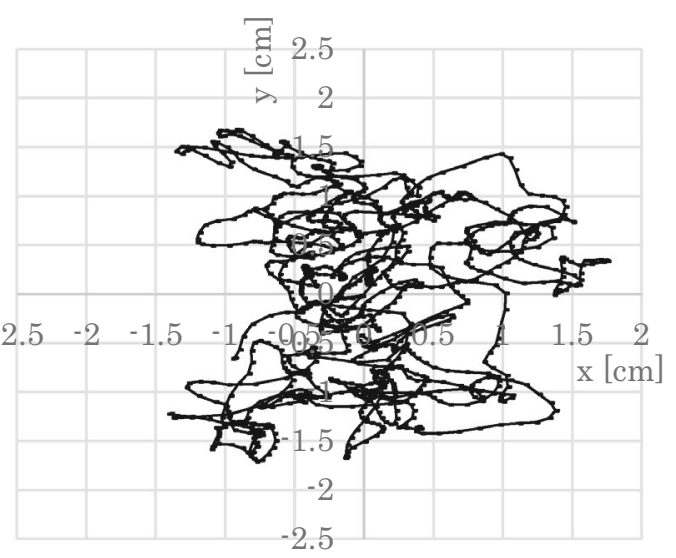

Fig. 3 Representative stabilograms recorded with the eyes closed. The control stabilogram (a) was compared to stabilograms recorded while subjects peripherally viewed 2D (b) and 3D (c) video clips, and while visually tracking 2D (d) and 3D (e) video clips

$(p<0.05)$. While the main effect of the vision method also tended to be seen in the sub-score $\mathrm{D}(p<0.1)$, the main effect of solidity of the vision was observed in this subscore $(p<0.05)$. With reference to the VAS, the subjects complained of eye strain after peripheral viewing of the video clips, which was indicated by the main effect of the vision method in this subjective evaluation $(p<0.01)$.
Lens accommodation and convergence distance

The measurements for the participants showed roughly similar results. For 3D vision, typical results for a participant (38-year-old male, emmetropia) are shown in Fig. 6. Fixation distances among accommodation, convergence, and depth map (theoretical distance of the surfaces of scene 

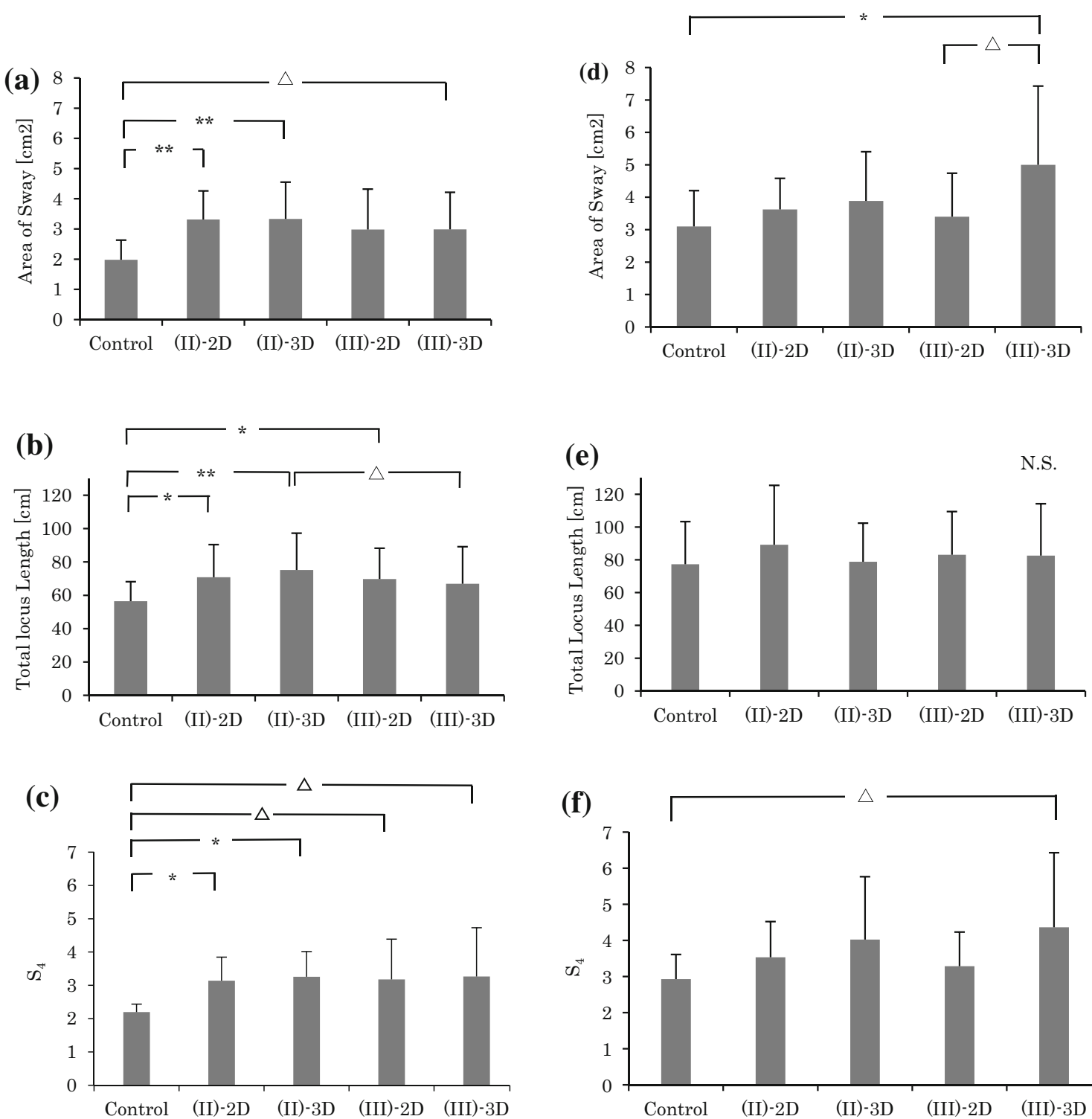

Fig. 4 Results of post hoc analysis for Friedman ANOVA. The sway values while the eyes were open $(\mathbf{a}, \mathbf{b}, \mathbf{c})$ and closed $(\mathbf{d}, \mathbf{e}, \mathbf{f})$ were compared. a, b, c show the sway values calculated from stabilograms

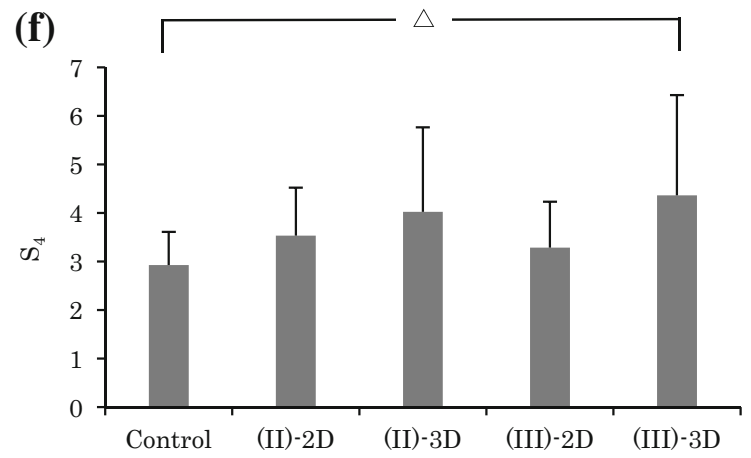

during exposure to video clips. The vertical axis corresponds to the area of sway in $(\mathbf{a}, \mathbf{d})$, total locus length in $(\mathbf{b}, \mathbf{e})$, and sparse density $\mathrm{S}_{4}$ in (c, f). $* p<0.05 ;{ }^{\triangle} p<0.1$

objects from a viewpoint) are compared while participants viewed the (II)-3D video clips on the LCD with repeated measures. Disagreement between the visual accommodation and convergence was not seen for the first $60 \mathrm{~s}$ of continuous viewing of the 3D images (Fig. 6). Consistency was not observed, and we were able to detect a delay in accommodation variations.

\section{Discussion}

In this paper, we examined whether motion sickness could be induced by the exposure to stereoscopic video clips, and

could worsen according to the vision method. Solidity of the subjects' vision (2D/3D) and their vision method (visual pursuit/peripheral viewing) were assumed to be influencing factors, which were examined using the two-way ANOVA. With eyes open, main effects of these factors were not obtained for any sway values although most of sway values were greater than the control during viewing of $2 \mathrm{D} / 3 \mathrm{D}$ video clips. We also recorded stabilograms with eyes closed, immediately after the exposure to the video clips (Fig. 3). Findings related to area of sway and sparse density of high degree showed a consistent tendency in main effect of the solidity. Further, after the peripheral viewing of the $3 \mathrm{D}$ video clip, remarkable change was seen in the sway 

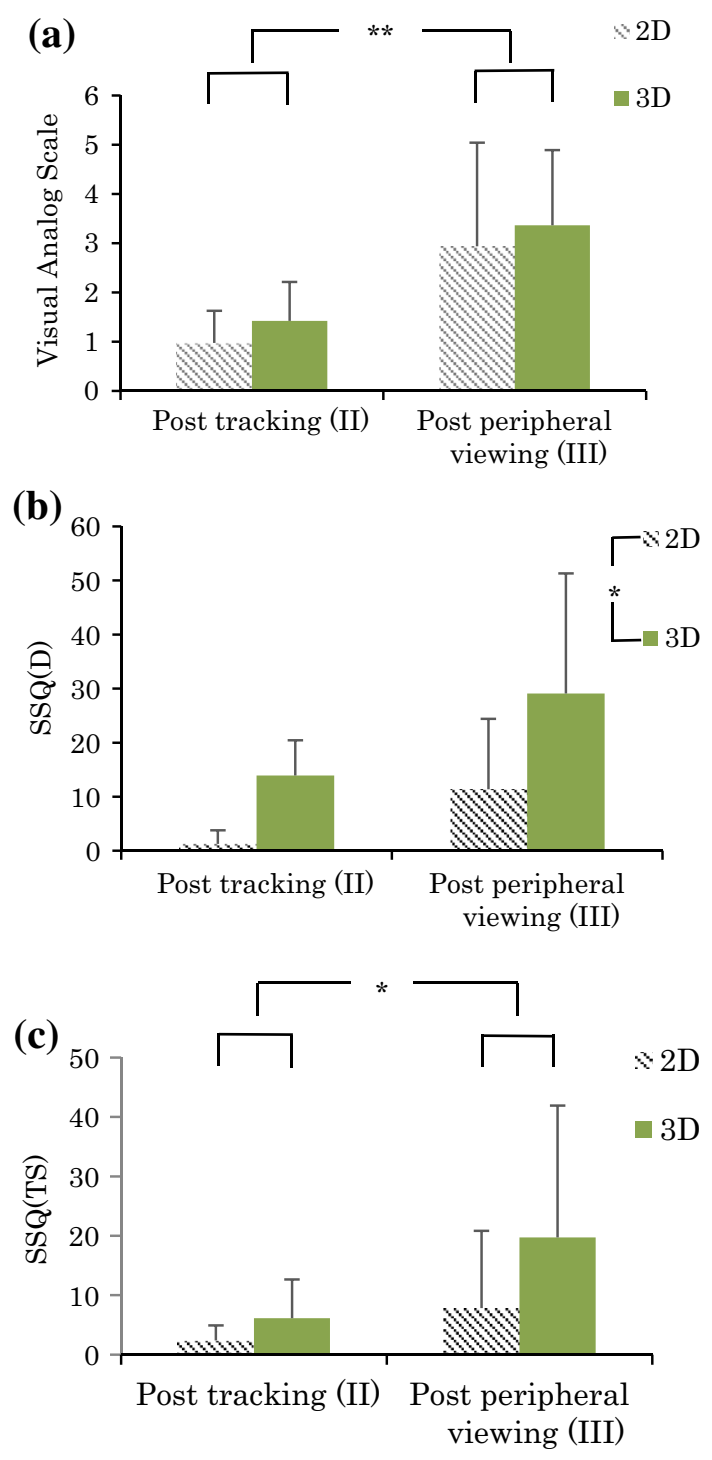

Fig. 5 Results of two-way ANOVA for subjective evaluation using the visual analog scale (a), SSQ-D measuring disorientation (b), and SSQ-TS measuring the total score (c)

values, thus explaining the instability in the subjects' equilibrium function. The change was also obtained from the statistical results of subjective evaluation after peripherally viewing the video clips. According to the analysis of the SSQ sub-score N, OD, TS, and VAS, we could found increase in severity of the symptoms such as uncomfortable and eye strain that were induced by the VIMS.

With eyes open, the total locus length significantly increases with visual pursuit to the stereoscopic sphere, which was not seen in the sway value immediately after the exposure to the video clip (Fig. 4b, e). Therefore, this influence is considered to be temporary. In contrast, instability of our equilibrium systems could be detected by the analysis of sway values with difficulty while peripherally viewing video clips (Fig. 4a, b, c). With eyes closed,

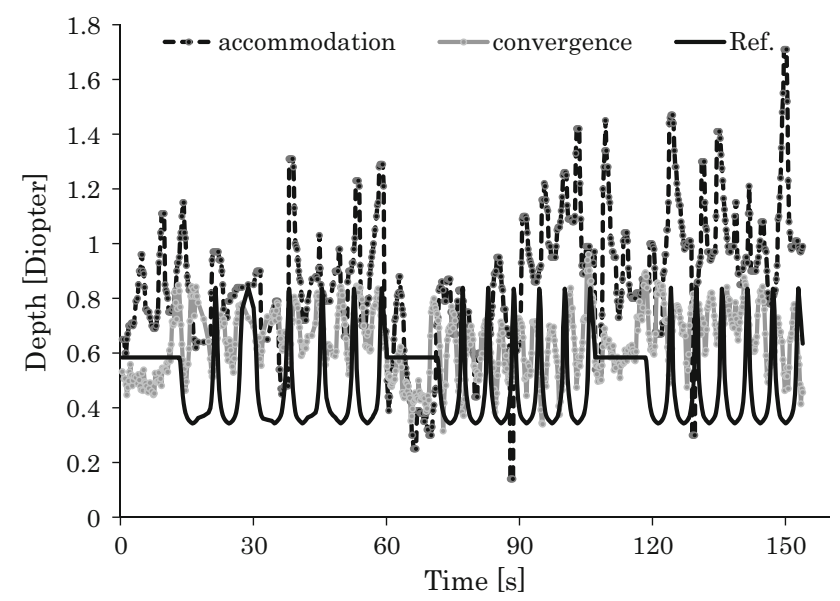

Fig. 6 Results of simultaneous measurement of lens accommodation and convergence distance while subjects viewed the (II)-3D video clips for 160 s. Dark continuous line indicates theoretical distance of the surfaces of scene objects from subjects' viewpoint (depth map)

however, significant increase was not only seen in most of the sway values including the previous indices for stabilograms, but also in the subjective evaluations including the eye strain component (Fig. 5). This is regarded as persistent influence which might be caused by peripheral viewing in unskillful background elements because their depth perception differs from our experience in daily life. This indicates that a labored style of visual performance could be induced by the binocular parallax because the stereoscopic images were constructed without consideration of our finite convergence. From this point of view, technology to compose stereoscopic images has been developed as a countermeasure [21], and is already being used in the following accommodation training. This technology is expected to be widely accepted. We used this technology in an accommodation training study on preventive medicine, which aimed to improve the pseudomyopia by relaxing the contracted focus-adjustment muscles around the eyeball, such as the ciliary and extraocular muscles [22]. We verified the short-term effects of the apparatus on the eyesight of visual inspection workers (22 females) suffering from eye fatigue. The workers were trained in 3 days. We found that the visual acuity of the subjects was statistically improved by continuous accommodation training, which will promote a ciliary musclestretching effect.

However, it is still unclear how long the duration of the impact of the mild symptoms induced by peripherally viewing of the $3 \mathrm{D}$ video clips on our equilibrium systems continues to remain. To approach the mechanism, we investigated the relationship among the body sway, visual function, and head posture while viewing stereoscopic video clips. Until recently, it has been believed that our convergence adjusts to virtual depth popped up from 3D 
TV screen, whereas human beings focus on the surface of the display during stereoscopic vision. It is generally explained that, "During the stereoscopic vision, accommodation and convergence are mismatched, and this is the main reason for the visual fatigue caused by viewing $3 \mathrm{D}$ video clips." According to the findings presented in our previous report [23], however, such explanations are incorrect. We developed a new device that can simultaneously measure accommodation and convergence. As a result, lens accommodation is considered to be consistent with convergence because the accommodation does not adjust to the surface of the display, but adjusts to the virtual depth during the stereoscopic vision [24].

Wann et al. [8] stated that within a virtual reality system, the eyes of a subject must maintain accommodation at the fixed LCD screen, despite the presence of disparity cues that necessitate convergence eye movements to capture the virtual scene. Moreover, Hong et al. [25] stated that the natural coupling of eye accommodation and convergence while viewing a real-world scene is broken when viewing stereoscopic displays. More recently, Patterson [26] provided an estimate of the depth of field of the human eye, and showed that an accommodation-vergence conflict is unlikely to occur under most stereo display viewing conditions, i.e., it might occur only with near eye displays. The critical issue is to present the virtual images at depth planes that fall within the depth of the field of the viewer, which would prevent such conflict, and which is likely to happen often, under most conditions. When the virtual image is presented within the depth of the field, accommodation can follow the vergence response without any conflict, because there would be no blur signal to drive accommodation back to the display surface. Under such conditions, it could be predicted that the viewer would accommodate at the distance of the convergence response, for which support is provided, as demonstrated in our previous studies [23, 27, 28]. Moreover, Patterson and Silzars [29] proposed that the eye strain and viewing discomfort that accompany viewing of stereo displays come from the high-level cue conflict between the presence of binocular parallax in the display and the absence of motion parallax.

We can stereoscopically view parallax images with operation of retinal/sensory fusion and vergence/motor fusion. We could succeed in binocular single vision by the convergence adjustment only if the binocular parallax were set such that it exceeds the limit of the retinal/sensory fusion. In the case of a large parallax, it is impossible to stereoscopically view the images with these operations. While stereoscopically viewing the images, we always conduct these two adjustments to help us focus on images popping out of the screen. The virtual images would be blurred if this adjustment was difficult. During this, stereoscopic vision causes uncomfortable symptoms induced by the VIMS, such as intoxication and eye strain. Nagata [30] investigated individual differences in the function of the vergence/motor fusion for stereoscopic vision, to suggest design criteria for safe 3D images for most of Japanese subjects, that did not get blurred. We need to now proceed by focusing on images popping out of the screen, to prevent the emergence of the VIMS symptoms caused by the exposure to blurred virtual images.

In a previous study, we also compared fixation distances between accommodation and convergence in middle-aged subjects while they followed a sphere on 2D/3D video clips used in the present study [31]. The measurements for the subjects showed roughly similar results. Fixation distances among accommodation, convergence, and depth map (theoretical distance of the surfaces of scene objects from a viewpoint) were compared while the subjects viewed the (II)-3D video clips on the LCD, with repeated measures. Disagreement between the visual accommodation and convergence was not seen for the first $60 \mathrm{~s}$ of continuously viewing the $3 \mathrm{D}$ images. However, gradually, their inconsistency was observed in Fig. 6, and we could find the delay in accommodation variations. The results indicated that subjects' accommodation and convergence when viewing $3 \mathrm{D}$ images were found to change the diopter value, which was synchronous to the movement of the 3D images. However, the lens accommodation was not consistent with the convergence in the middle-aged subjects after gazing at the 3D video clips for $90 \mathrm{~s}$. However, subjects in the present study did not follow the stereoscopic sphere for more than $90 \mathrm{~s}$, and the abovementioned inconsistency was not induced considerably. This indicates that the inconsistency between the lens accommodation and convergence may not cause the 3D sickness. Moreover, we herein speculate the mechanism of temporal increase in the body sway value observed with visual pursuit of virtual objects. Conceivably, our head posture would be synchronized to pursue virtual objects and become unstable. Instability of the head posture might enhance the sway value because the body sway is affected by the head posture.

To enhance labor effectiveness, wearable apparatus such as cameras and head-mounted displays have been recently introduced to the industrial field. On the other hand, one major limitation of this work is the small sample. Gender and age are also a limiting factors. Generally speaking, sway disturbance may occur frequently in women and older adults. Systems to control upright posture are actually considered less stable in older adults than in young individuals [32]. This research targeted more stable young men and examined whether the 3D disturbance of sway could be detected. We hope to extend this work to participants of other gender and age groups in the industrial field, including children who often play video games. 
Vision plays an important role of facilitating the input of about $80 \%$ of ambient information in our central nervous system [33]. Therefore, the visual deprivation and adaptation to luminosity considerably depend on the stability of biological control systems. The visual information also depends on the system to control upright posture. In this study, the influence of the vision method, i.e. visual pursuit and peripheral viewing, on our equilibrium systems during and after viewing 2D/3D video clip, was examined. We found that irrespective of the vision method followed by the subject, the sway values were greater than the control during viewing of $2 \mathrm{D} / 3 \mathrm{D}$ video clips. Systems to control their upright posture tended to become unstable during exposure to the video clips. In addition to deterioration in their equilibrium function, subjective acute exacerbation was observed after peripheral viewing of the video clips, in comparison with that after viewing clips involving visual pursuit of the sphere. This is regarded as persistent influence which might be caused by peripheral viewing in an unskillful background element and depth perception which differs from our experience in daily life. In future research, we will examine whether the severity of the VIMS depends upon exposure time, differential rate of backgrounds, and the vestibular input, and discuss limits of our ability in visual information and the mechanism of the VIMS.

Acknowledgments The authors would like to thank Olympus Memory Works, Inc., for their beautiful 3D video clips. This work was supported in part by the Ministry of Education, Culture, Sports, Science and Technology, Grant-in-Aid for Scientific Research (B) Number 24300046, (C) Number 26350004, and the Hori Information Science Promotion Foundation.

Ethical approval The research was approved by the Ethics Committee, Nagoya City University Graduate School of Natural Sciences: Project ID Number 42.

Conflict of interest The authors have no conflict of interest.

\section{Appendix: Sparse density}

For the data analysis, the anterior-posterior direction was considered to be independent of the lateral direction [35]. Stochastic differential equations (SDEs) were proposed as mathematical models to generate the stabilograms [6, 36, 37]. The variance in the stabilogram depends on the form of the temporally averaged potential function in the SDE, which generally has plural minimal points. In the vicinity of these points, local stable movement with a high-frequency component was generated as a numerical solution to the SDE. Therefore, we can expect a high density of observed COP in this area on the stabilogram [6]; sparse density (SPD) is regarded as an index for this measurement.
Sparse density is defined by an average of the ratio $G_{j}(1) / G_{j}(k)$ for $j=3,4, \ldots, 20$, where $G_{j}(k)$ is the number of divisions having more than $k$ measured points. A stabilogram is divided into quadrates whose side length is $j$ times longer than the resolution. If the center of gravity is stationary, the SPD value is 1 . If there are variations in the stabilograms, the SPD value is greater than 1. Thus, the SPD depends on the characteristics of the stabilogram and the minimal structure of the temporally averaged potential function.

\section{References}

1. Malik AS, Choi TS, Nisar H (editors). Depth map and 3D imaging applications: algorithms and technologies. Hershey, PA, USA, Proc IGI Global Hershey. 2011.

2. Okawa T, Tokita T, Shibata Y, Ogawa T, Miyata H. Stabilometry: significance of locus length per unit area (L/A) in patients with equilibrium disturbances. Equilib Res. 1995;55:283-93 (in Japanese).

3. Kaga K, Memaino K. Structure of vertigo. Tokyo: Kanehara; 1992 (in Japanese).

4. Okawa T, Tokita T, Shibata Y, Ogawa T, Miyata H. Stabilometry: significance of locus length per unit area (L/A). Equilib Res. 1995;54:296-306 (in Japanese).

5. Takada H, Kitaoka Y, Ichikawa S, Miyao M. Physical meaning on geometrical index for stabilometry. Equilib Res. 2003;62:168-80 (in Japanese).

6. Takada H, Kitaoka Y, Shimizu Y. Mathematical index and model in stabilometry. Forma. 2001;16:17-46.

7. Takada H, Fujikake K, Miyao M, Matsuura Y. Indices to detect visually induced motion sickness using stabilometry. In: Proc VIMS. 2007. p. 178-83.

8. Wann J, Rushton S, Mon Williams M. Natural problems for stereoscopic depth perception in virtual environments. Vis Res. 1995;35:2731-6.

9. Yasui R, Matsuda I, Kakeya H. Combining volumetric edge display and multiview display for expression of natural $3 \mathrm{D}$ images. Proc SPIE. 2006;6055:0Y1-9.

10. Kakeya H. MOEVision: simple multiview display with clear floating image. Proc SPIE. 2007;6490:64900J.

11. Kennedy RS, Lane NE, Berbaum KS, Lilienthal MG. A simulator sickness questionnaire (SSQ): a new method for quantifying simulator sickness. Int J Aviat Psychol. 1993;3:203-20.

12. Holomes SR, Griffin MJ. Correlation between heart rate and the severity of motion sickness caused by optokinetic stimulation. J Psychophysiol. 2001;15:35-42.

13. Himi N, Koga T, Nakamura E, Kobashi M, Yamane M, Tsujioka $\mathrm{K}$. Differences in autonomic responses between subjects with and without nausea while watching an irregularly oscillating video. Auton Neurosci. 2004;116:46-53.

14. Yokota Y, Aoki M, Mizuta K. Motion sickness susceptibility associated with visually induced postural instability and cardiac autonomic responses in healthy subjects. Acta Otolaryngol. 2005; $125: 280-5$.

15. Scibora LM, Villard S, Bardy B, Stoffregen TA. Wider stance reduces body sway and motion sickness. In: Proc VIMS. 2007. p. $18-23$.

16. Fujikake K, Miyao M, Watanabe T, Hasegawa S, Omori M, Takada H. Evaluation of body sway and the relevant dynamics 
while viewing a three-dimensional movie on a head-mounted display by using stabilograms. In: Shumaker R, editor. Lecture notes in computer science. Berlin, Heidelberg: Springer; 2009. p. $41-50$.

17. Takada M, Murakami K, Kunida Y, Hirata T, Matsuura Y, Iwase $\mathrm{S}$, et al. Effect of hour-long stereoscopic film on equilibrium function. In: Proc IMID Digest. 2011. p. 737-38.

18. Yoshikawa K, Takada H, Miyao M. Effect of display size on body sway in seated posture while viewing an hour-long stereoscopic film. In: Stephanidis C, Antona M, editors. UAHCI/HCII 2013 part II, LNCS 8010. Berlin, Heidelberg: Springer; 2013. p. $336-41$.

19. Suzuki J, Matsunaga T, Tokumatsu K, Taguchi K, Watanabe I. Q \& A and a manual in stabilometry. Equilib Res. 1996;55:64-77 (in Japanese).

20. Hollander M, Wolfe DA. Nonparametric statistical methods. New York-Sydney-Tokyo-Mexico City: Wiley; 1973.

21. Nishihira T, Tahara $\mathrm{H}$. Apparatus for recovering eyesight utilizing stereoscopic video and method for displaying stereoscopic video. US. Patent. 2002;US7404693B2.

22. Takada M, Miyao M, Satoh M, Yoshikawa K, Matsuura Y, Takada $\mathrm{H}$. Effect of accommodation training on visual function of visual inspection workers and middle-aged people. J Sports Med Doping Stud. 2012; doi:10.4172/2161-0673.1000112.

23. Miyao M, Ishihara S, Saito S, Kondo T, Sakakibara H, Toyoshima $\mathrm{H}$. Visual accommodation and subject performance during a stereographic object task using liquid crystal shutters. Ergonomics. 1996;39:1294-309.

24. Shiomi T, Uemoto K, Kojima T, Sano S, Ishio H. Simultaneous measurement of lens accommodation and convergence in natural and artificial 3D vision. J Soc Inf Disp. 2013;21:120-8.

25. Hong H, Sheng L. Correct focus cues in stereoscopic displays improve 3D depth perception. SPIE Newsroom. 2010;. doi:10. 1117/2.1201007.003109.

26. Patterson R. Human factors of stereo displays: an update. J Soc Inf Disp. 2009;17:987-96.
27. Miyao M, Shiomi T, Hori H, Hasegawa A, Matsuura Y, Takada $\mathrm{H}$, et al. Comparison of $2 \mathrm{D}$ and $3 \mathrm{D}$ vision gaze with simultaneous measurements of accommodation and convergence among young and middle-aged subjects. In: Proc IMID Digest. 2011. p. 149-50.

28. Kojima T, Matsuura Y, Miyao M, Shiomi T, Takada H. Comparison by simultaneous measurement of lens accommodation and convergence in 3D vision and their distributions. Int $\mathrm{J}$ Biosci Biochem Bioinforma. 2013;3:635-8.

29. Patterson R, Silzars A. Immersive stereo displays, intuitive reasoning, and cognitive engineering. J Soc Inf Disp. 2009;17:443-8.

30. Nagata S. Distribution of "vergence fusional stereoscopic limits (VFSL)" of disparity in a stereoscopic display. TVRSJ. 2002;7:239-46 (in Japanese).

31. Takada M, Uemoto K, Miyao M, Matsuura Y, Satoh M, Takada $\mathrm{H}$. Comparison of fixation distances in middle-aged subjects. Int $\mathrm{J}$ Biosci Biochem Bioinforma. 2013;2:389-94.

32. Takada H, Kitaoka Y, Iwase S, Shimizu Y, Watanabe T, Nakayama M, Miyao M, Mihashi K. Characteristic changes of sway of center of gravity with advancing age. Environ Med. 2004;47:85-9.

33. Kato Z, Okubo T. Methods to measure bio functions for beginners. Tokyo: Nihon Shuppan Service Co, Ltd; 1999 (in Japanese).

34. Sugiura A, Miyao M, Yamamoto T, Takada H. Effect of strategic accommodation training by wide stereoscopic movie presentation on myopic young people of visual acuity and asthenopia. Displays. 2011;32(4):219-24.

35. Goldie PA, Bach TM, Evans OM. Force platform measures for evaluating postural control: reliability and validity. Arch Phys Med Rehabil. 1986;70:510-7.

36. Collins JJ, De Luca CJ. Open-loop and closed-loop control of posture: a random-walk analysis of center of pressure trajectories. Exp Brain Res. 1993;95:308-18.

37. Newell KM, Slobounova SM, Slobounov ES, Molenaar PC. Stochastic processes in postural center-of-pressure profiles. Exp Brain Res. 1997;113:158-64. 